\title{
Catastrophic/benign flutter boundary evaluation carried for two-dimensional aerodynamic surface in subsonic flow
}

\author{
Wenjia Cai $^{1, a}$, Laith K. Abbas ${ }^{1, b *}$, Dongyang Chen ${ }^{1, c}$, Xiaoting Rui ${ }^{1, d}$, \\ Pier Marzocca ${ }^{2, e}$ \\ ${ }^{1}$ Institute of Launch Dynamics, Nanjing University of Science and Technology, Nanjing, 210094, \\ Jiangsu, China \\ ${ }^{2}$ School of Engineering, Aerospace Engineering and Aviation Discipline, RMIT University, Melbourne, \\ VIC 3000, Australia \\ a1034282168@qq.com, ${ }^{b}$ laithabbass@yahoo.com ,ccdy_1988@sina.cn, 'ruixt@163.net, \\ epier.marzocca@rmit.edu.au
}

Keywords: Lyapunov first quantity; Aeroelastic flutter; Transfer matrix method; Computational fluid dynamics; Limit cycle oscillation.

\begin{abstract}
The catastrophic/benign flutter boundary character evaluation and its control are carried out for 2-DOF lifting surfaces. Catastrophic/benign implies that the Limit Cycle Oscillation (LCO) is either unstable or stable. Within this work: (1) Quasi-Steady (QS) theory and Computational Fluid Dynamics (CFD) for subsonic flow calculations are implemented, (2) Flutter motion equations of a two-dimensional airfoil section with cubic nonlinear stiffness in the pitching direction are established, (3) Uncoupled bending and torsion frequencies of the selected aerodynamic surface are computed using recently developed Transfer Matrix Method of Multibody Systems (MSTMM), and (4) Lyapunov's First Quantity (LFQ) is computed to study the bifurcation behavior of the aeroelastic system in the vicinity of the flutter boundary. The main objective of this study is to implement a control capability enabling one to control both the flutter boundary and its character as to safely expand the operational envelop of the aerospace vehicle without failure.
\end{abstract}

\section{Introduction}

One of the major challenges facing aeronautical and space vehicles designers today is that of aeroelasticity as shown in Fig. 1. Complex interactions between dynamics, solid mechanics, and aerodynamic forces can create problems if not well understood and analyzed. Aerospace vehicles structural fatigue, passenger discomfort, decreased performance, and even catastrophic failure can result. Today's aerospace vehicles, specifically aircraft, however, are expected to push the physical limits in terms of speed, altitude, maneuverability, endurance, range, and cost [1]. Designers are turning to lightweight materials for use with high-powered engines to reduce weight in order to carry

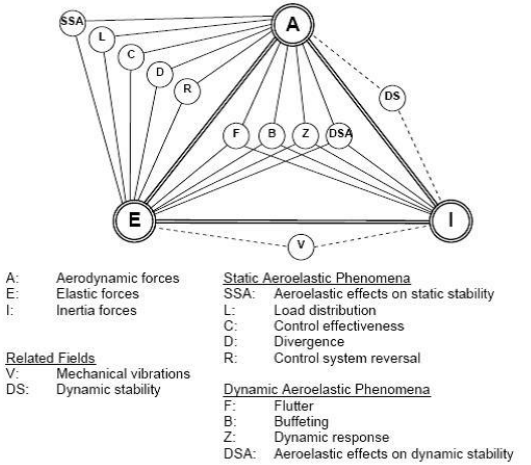

Figure 1. The aeroelastic traingle of forces

more fuel and payload. These lightweight materials exhibit more flexibility than conventional aircraft/missiles materials which when used at higher speeds and altitudes pose possible aeroelastic concerns. Aeroelasticity can be broken into two main categories: static and dynamic. Cases in which the inertial forces play a negligible role are referred to as static. Dynamic aeroelasticity involves influential inertial forces and the associated instabilities are referred to as flutter. Flutter is a dynamic instability phenomenon resulting from the interaction between an elastic structure and the flow around the structure. Depending on the nature of the flutter boundary, i.e. catastrophic or benign, if the vehicle reaches the flutter speed it can feature a catastrophic failure (unstable limit cycle 
oscillations (LCO)) or can survive (stable LCO), respectively. In the latter case, the failure will not occur catastrophically, but by fatigue [2]. Therefore, a better understanding of all the factors contributing to the occurrence of flutter and the character of the flutter instability are highly recommended. In addition, the possibility of controlling this issue is important. The goal of the control is to expand the flight envelope without weight penalties by increasing the flutter speed and to convert the catastrophic flutter into benign one [3]. The principle of catastrophic and benign types of flutter can be found in the different literature under different terminologies that depend on the particular approach of the problem itself. In this study, the determination of the catastrophic/benign character of the flutter boundary and its control is carried out via determination of the sign of the Lyapunov First Quantity (LFQ) [4], for the flutter boundary that corresponds to the purely imaginary roots of the characteristic equation.

Multibody system dynamics (MSD) has become an important theoretical tool for wide engineering problems analysis. Several MSD methods have been studied both theoretically and computationally. Rui and colleagues [5-9] transferred the concept of State Vectors (SVs) into the classical transfer matrix method, and built up a new multibody dynamics method called Transfer Matrix Method of Linear Multibody Systems (MSTMM). It is a new efficient method where the overall transfer equations (TE) of the system are used in the transfer matrix method instead of the global dynamics equations of the system which is used in traditional dynamics method such as Finite Element Method (FEM).

The focus of this paper is to investigate the aeroelastic behavior of a two-dimensional aerodynamic surface (for airplane wings such as vertical and horizontal stabilizers (Fig. 2(a)) and for missile such as flight control surfaces (Fig. 2(b)). Quasi-steady (QS) theory and Computational Fluid Dynamics (CFD) are used for aerodynamic modeling. A two-degrees-of-freedom (2-DOF) airfoil section with cubic nonlinear stiffness in pitching direction is theoretically modeled. Uncoupled bending and torsion frequencies for the selected aerodynamic surface are computed using MSTMM. Lyapunov first quantity is used to the determination of the catastrophic/benign character of the flutter boundary.

(a)

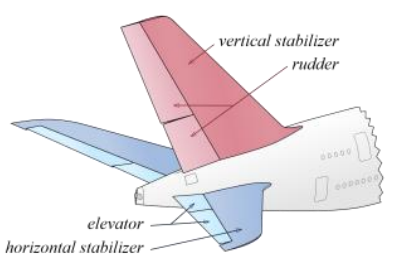

Figure 2. (a) Vertical and horizontal stabilizers on the airplane, and (b) Four main categories of missile flight controls

\section{Aeroelastic Equations of Motion}

\section{$2.1 \quad$ Structural model}

The aerodynamic surface of semi-span $L$ in Fig. 3 (a) is modeled as a chordwise rigid (Section A-A in Fig. 3 (a)), and the two-degrees-of-freedom (2-DOF) airfoil model that accommodates motion in pitch ( $\alpha$, positive nose-up) and plunge ( $h$, positive down) immersed in a horizontal flow of undisturbed speed $U_{\infty}$ is shown in Fig. 3 (b). The model is referred to in the literature as the typical section. Control surfaces are connected to the main body via torsionally less stiff shafts as sketched in Fig. 3 (c). For purposes of theoretical flutter prediction, inertia and geometric properties of a lifting surface/or control surface can be represented by a typical section with inertia and geometric properties of the surface at $3 / 4$ of the distance from the root of the wing [10]. The airfoil can pitch about an elastic axis which is defined as being perpendicular to the shear center of the airfoil. The structural stiffness in plunge is modeled with linear spring coefficient $K_{h}$, while the restoring moment is modeled with linear and nonlinear torsional

(a)

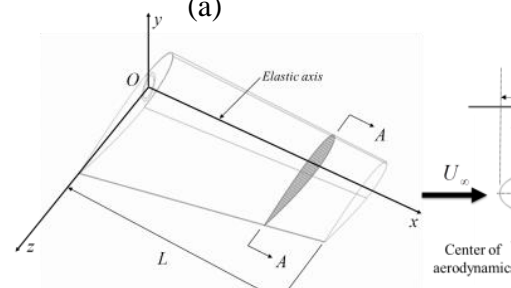

Figure 3. (a) Sketch of aerodynamic surface, (b) 2-DOF airfoil model (section A-A), and (c) Control surface with shaft, which modeled as a torsional spring 
stiffness coefficients $K_{\alpha}$ and $\hat{K}_{\alpha}$, respectively. Noteworthy points on the airfoil include the center of gravity, elastic axis, and aerodynamic center. The non-dimensional distances (as a fraction of $b$ and positive in the positive $z$-direction) from the elastic axis (center of rotation) to the center of gravity and to the mid-chord position are denoted as $x_{\alpha}=S_{\alpha} / m b$ and $a$, respectively, where $S_{\alpha}$ is the static unbalance about the elastic axis. The 2-DOF nonlinear (autonomous ordinary differential equations $(\mathrm{ODE}))$ aeroelastic governing equations can be written as $[3,11]$

$$
\begin{aligned}
& m \ddot{h}(\mathrm{t})+S_{\alpha} \ddot{\alpha}(\mathrm{t})+c_{h} \dot{h}(\mathrm{t})+k_{h} h(\mathrm{t})=-F_{\text {aero }}^{h}(t) . \\
& S_{\alpha} \ddot{h}(\mathrm{t})+I_{\alpha} \ddot{\alpha}(\mathrm{t})+c_{\alpha} \dot{\alpha}(\mathrm{t})+k_{\alpha} \alpha(\mathrm{t})+\delta_{S} \hat{k_{\alpha}} \alpha^{3}(\mathrm{t})=F_{\text {aero }}^{\alpha}(t)-F_{C}^{\alpha} .
\end{aligned}
$$

In Eq. (1), $m$ is the airfoil mass per unit length, $I_{\alpha}=m b^{2} r_{\alpha}^{2}$ is the airfoil mass moment of inertia about the elastic axis per unit length, $r_{\alpha}^{2}$ is the radius of gyration about elastic axis, $c_{h}$ and $c_{\alpha}$ are plunge and pitch viscous damping coefficient, respectively, $F_{\text {aero }}^{h}, F_{\text {aero }}^{\alpha}$ and $F_{C}^{\alpha}=\sigma_{1} \alpha(t)+\delta_{C} \sigma_{2} \alpha^{3}(t)$ are lift (positive downward), moment (positive leading edge up), and the active nonlinear control, where $\sigma_{1}, \sigma_{2}, \delta_{S}$ and $\delta_{C}$ are respectively, the linear, nonlinear control gains and tracers can take the value 1 or 0 depending on whether the nonlinearity effect of structure/control is included or ignored.

\subsection{Aerodynamic model in subsonic flow}

For aeroelastic analyses, there are many of aerodynamic models starting from quasi-steady (QS) to highly complex numerical flow field solvers, such as 'Navier-Stokes (NS)' unsteady codes. However, applying complex CFD codes are computationally expensive, quite long to set up as well and required huge memory although still cheaper than measuring the aerodynamic characteristics of an aerospace vehicle in a wind tunnel [12]. The QS aerodynamic approximations are widely used in the pre-design stages, as the simplest aerodynamic modeling technique. In the following, the QS aerodynamic lift force $F_{\text {aero }}^{h}$ and moment $F_{\text {aero }}^{\alpha}$ (with respect to the elastic axis) per unit span are

$$
\begin{aligned}
& F_{\text {aero }}^{h}(t)=\pi \rho_{\infty} b^{2} U_{\infty} \dot{\alpha}(\mathrm{t})+\rho_{\infty} U_{\infty}^{2} b C_{L}^{\alpha}\left(\alpha(\mathrm{t})+\frac{\dot{h}(\mathrm{t})}{U_{\infty}}+(1 / 2-a) \frac{b \dot{\alpha}(\mathrm{t})}{U_{\infty}}\right) . \\
& F_{\text {aero }}^{\alpha}(t)=-\pi \rho_{\infty} b^{2} U_{\infty} b(1 / 2-a) \dot{\alpha}(\mathrm{t})+\rho_{\infty} U_{\infty}^{2} b C_{L}^{\alpha}(1 / 2+a)\left(\alpha(\mathrm{t})+\frac{\dot{h}(\mathrm{t})}{U_{\infty}}+(1 / 2-a) \frac{b \dot{\alpha}(\mathrm{t})}{U_{\infty}}\right) .
\end{aligned}
$$

where $\rho_{\infty}$ and $C_{L}^{\alpha}$ are the air density and lift coefficient, respectively. Although the QS aerodynamic model is relatively simple, it is very useful and has proven to provide insight on the physical behavior of the aeroelastic system.

\subsection{Modeling of aerodynamic surface with shaft in the view of MSTMM methodology}

The aerodynamic surface connected with shaft shown in Fig. 3 (c) may model it as coupled bending-torsion Euler-Bernoulli beam with torsional spring in the view of MSTMM. The full theory of MSTMM is described in $[9,13]$. However, the SV of a connection point is given by kinematic and kinetic quantities in physical coordinates: in case of linear multibody systems, vibrations are described by small displacements $x, y, z$ along the Cartesian axes and small angular rotations $\theta_{x}, \theta_{y}, \theta_{z}$ about these axes; cutting forces and moments are given by $q_{x}, q_{y}, q_{z}$ and $m_{x}, m_{y}, m_{z}$, respectively. In 3-dimensional case (3D) with $n_{s}=12$ ( $n_{s}$ is the number of state variables in the SV), the SV in physical and modal coordinates at the connection point are summarized in a vector, receptively.

$\left.z\right|_{\text {physical coordinates }}=\left[x, y, z, \theta_{x}, \theta_{y}, \theta_{z}, m_{x}, m_{y}, m_{z}, q_{x}, q_{y}, q_{z}\right]^{T}$

$\left.Z\right|_{\text {modal coortinates }}=\left[X, Y, Z, \Theta_{x}, \Theta_{y}, \Theta_{z}, M_{x}, M_{y}, M_{z}, Q_{x}, Q_{y}, Q_{z}\right]^{T}$

The differential equation of the coupled (a)

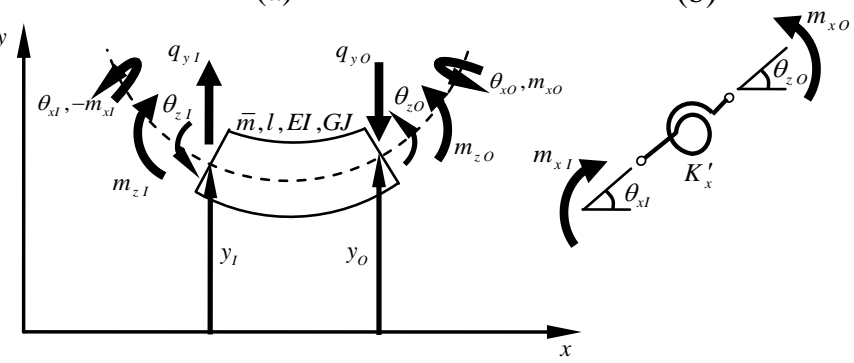

Figure 4.

Some typical elements in MSTMM

library: (a) Beam segment, and (b) Torsional spring 
bending-torsion Euler-Bernoulli beam vibrating in a plane with frequency $\omega$ shown in Fig. 4 (a) for MSTMM terminology with length $l$, bending stiffness $E I$, torsional rigidity $G J$, and its modal transformation are given as [14]

$$
\begin{aligned}
& \left.E I y^{I V}+m \ddot{y}-m \chi_{\alpha} b \ddot{\theta}_{x}=0\right\} \underset{y(x, t)=Y(x) \sin \omega t}{ } E I Y^{I V}(x)-m \omega^{2} Y(x)+m \chi_{\alpha} b \omega^{2} \Theta_{x}(x)=0 . \\
& \left.G J \theta_{x}^{I I}+m \chi_{\alpha} b \ddot{y}-I_{\alpha} \ddot{\theta}_{x}=0\right\} \stackrel{\frac{\partial}{\theta_{x}(x, t)=\theta_{x}(x) \sin \omega t}}{\longrightarrow} G J \Theta_{x}^{I I}(x)-\omega^{2} m \chi_{\alpha} b Y(x)+I_{\alpha} \omega^{2} \Theta_{x}(x)=0 .
\end{aligned}
$$

The subscripts $I$ and $O$ in Fig. 4 are element's input and output, respectively. Eliminating either $Y(x)$ or $\Theta_{x}(x)$ from Eq. (4), one equation in sixth order differential in non-dimensional form (non-dimensional length $\xi=x / l$ ) can be obtained

$$
\begin{aligned}
& W^{V I}+\left(I_{\alpha} \omega^{2} / G J\right) W^{I V}-\left(m \omega^{2} / E I\right) W^{I I}-\left(m \omega^{2} / E I\right)\left(I_{\alpha} \omega^{2} / G J\right) W\left(1-m \chi_{\alpha}^{2} b^{2} / I_{\alpha}\right) W=0 \\
& \text { or, } \quad\left(D^{6}+a D^{4}-b D^{2}-a b c\right) W=0 \text {; where } \quad a=I_{\alpha} \omega^{2} l^{2} / G J, b=m \omega^{2} l^{4} / E I, c=1-m \chi_{\alpha}^{2} b^{2} / I_{\alpha}, D=\mathrm{d} / \mathrm{d} \xi .
\end{aligned}
$$

The general solution of Eq. (5) can be cast as $W(\xi)=C_{1} \cosh \alpha \xi+C_{2} \sinh \alpha \xi+C_{3} \cos \beta \xi+C_{4} \sin \beta \xi+$ $C_{5} \cos \gamma \xi+C_{6} \sin \gamma \xi$ with arbitrary constant coefficients $C_{1}, \cdots C_{6} . W(\xi)$ represents the solution for both the bending displacement $Y$ and the torsional rotation $\Theta_{x}$ with different constant values. Thus $Y(\xi)=A_{1} \cosh \alpha \xi+A_{2} \sinh \alpha \xi+A_{3} \cos \beta \xi+A_{4} \sin \beta \xi+A_{5} \cos \gamma \xi+A_{6} \sin \gamma \xi$ and $\Theta_{x}(x)=B_{1} \cosh \alpha \xi+B_{2} \sinh \alpha \xi+$ $B_{3} \cos \beta \xi+B_{4} \sin \beta \xi+B_{5} \cos \gamma \xi+B_{6} \sin \gamma \xi$ where $A_{1}, \cdots A_{6}$ and $B_{1}, \cdots B_{6}$ are the two different sets of constants [14]. For the Euler-Bernoulli beam vibrating in a plane, the SV in Eq. (4) can be reduced to include $Y(\xi), \Theta_{x}(\xi)$, and the linearized relations $\Theta_{z}=Y^{\prime} / l, M_{z}=-E I Y^{\prime \prime} / l^{2}, Q_{y}=-M_{z}^{\prime} / l, M_{x}=-G J \Theta_{x}^{\prime} / l$ will lead to the modal solution functions of the TE $\boldsymbol{Z}(x)=\boldsymbol{B}(x) \boldsymbol{a}$ or

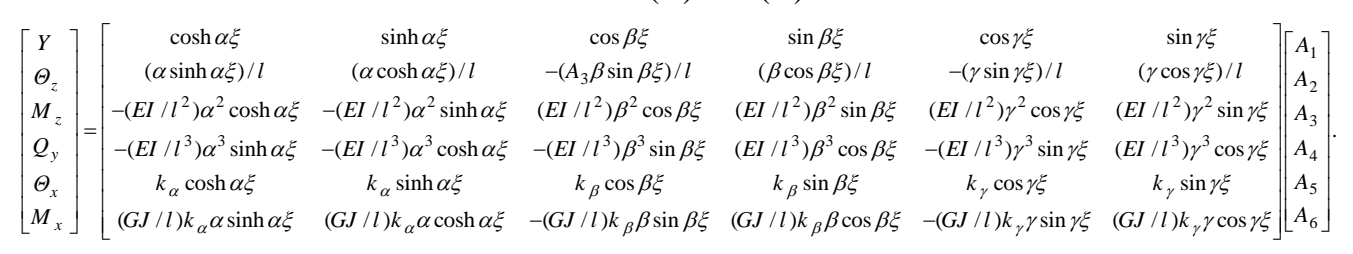

The coefficient vector $\boldsymbol{a}=\left[A_{1}, A_{2}, A_{3}, A_{4}, A_{5}, A_{6}\right]^{T}$ of unknown constants can be adopted as boundary condition $\boldsymbol{Z}_{I}$ at the input end such as $\boldsymbol{Z}_{I}=[\boldsymbol{B}(0)] \boldsymbol{a}$ or $\boldsymbol{a}=[\boldsymbol{B}(0)]^{-1} \boldsymbol{Z}_{I}$. For the beam output end at $x=l$ or $\xi=1$, from Eq. (6) the output condition can be found

$$
\boldsymbol{Z}_{O}=[\boldsymbol{B}(l)] \boldsymbol{a}=[\boldsymbol{B}(l)][\boldsymbol{B}(0)]^{-1} \boldsymbol{Z}_{I}=\boldsymbol{U}^{C B} \boldsymbol{Z}_{I} .
$$

where, $\boldsymbol{U}^{C B}=[\boldsymbol{B}(l)][\boldsymbol{B}(0)]^{-1}$ is the bending-torsion coupled beam transfer matrix. A massless linear torsional spring may vibrate in a plane as shown in Fig. 4 (b). The moments at the input and output ends are equal and given by the rotation angles as $m_{x O}=m_{x I}=K_{x}^{\prime}\left(\theta_{x O}-\theta_{x I}\right)$. Applying the transformations $\theta_{x}=\Theta_{x} \sin \omega t, m_{x}=M_{x} \sin \omega t$, results in the TE

$$
\left[\begin{array}{c}
\Theta_{x} \\
M_{x}
\end{array}\right]_{O}=\left[\begin{array}{cc}
1 & 1 / K_{x}^{\prime} \\
0 & 1
\end{array}\right]\left[\begin{array}{c}
\Theta_{x} \\
M_{x}
\end{array}\right]_{I} \text { or } \quad \boldsymbol{Z}_{O}=\boldsymbol{U}^{T S} \boldsymbol{Z}_{I} \text { where } \boldsymbol{U}^{T S}=\left[\begin{array}{cc}
1 & 1 / K_{x}^{\prime} \\
0 & 1
\end{array}\right] .
$$

where $\boldsymbol{U}^{T S}$ can be written with $\mathrm{SV}$ in a form $\boldsymbol{Z}=\left[Y, \Theta_{z}, M_{z}, Q_{y}, \Theta_{x}, M_{x}\right]^{T}$.

\subsection{State-space form}

Introducing the following dimensionless variables: $\xi=h / b, k_{h}=m \omega_{h}^{2}, k_{\alpha}=m r_{\alpha}^{2} \omega_{\alpha}^{2}$, $V=U_{\infty} /\left(\omega_{\alpha} b\right), \zeta_{\alpha}=c_{\alpha} /\left(2 \sqrt{m r_{\alpha}^{2} k_{\alpha}}\right), \zeta_{h}=c_{h} /\left(2 \sqrt{m k_{h}}\right), \bar{\omega}=\omega_{h} / \omega_{\alpha}, \tau=U_{\infty} t / b, \Xi=\hat{k}_{\alpha} / k_{\alpha}, \Omega_{1}=\sigma_{1} / k_{\alpha}, \Omega_{2}=\sigma_{2} / k_{\alpha}$, $\mu=m / \pi \rho_{\infty} b^{2}$ and letting $\xi=x_{1}, \alpha=x_{2}, \xi^{\prime}=x_{3}$, and $\alpha^{\prime}=x_{4}$, or $\left(x=\left(\xi, \alpha, \xi^{\prime}, \alpha^{\prime}\right)^{T}=\left(x_{1}, x_{2}, x_{3}, x_{4}\right)^{T}\right)$, Eqs. (1 and 2) may written in the form of state-space [4]

$$
\begin{aligned}
& d x_{1} / d \tau=a_{1}^{(1)} x_{1}(\tau)+a_{2}^{(1)} x_{2}(\tau)+a_{3}^{(1)} x_{3}(\tau)+a_{4}^{(1)} x_{4}(\tau), \\
& d x_{2} / d \tau=a_{1}^{(2)} x_{1}(\tau)+a_{2}^{(2)} x_{2}(\tau)+a_{3}^{(2)} x_{3}(\tau)+a_{4}^{(2)} x_{4}(\tau), \\
& d x_{3} / d \tau=a_{1}^{(3)} x_{1}(\tau)+a_{2}^{(3)} x_{2}(\tau)+a_{222}^{(3)} x_{2}^{3}(\tau)+a_{3}^{(3)} x_{3}(\tau)+a_{4}^{(3)} x_{4}(\tau), \\
& d x_{4} / d \tau=a_{1}^{(4)} x_{1}(\tau)+a_{2}^{(4)} x_{2}(\tau)+a_{222}^{(4)} x_{2}^{3}(\tau)+a_{3}^{(4)} x_{3}(\tau)+a_{4}^{(4)} x_{4}(\tau) .
\end{aligned}
$$

Let $S_{N L}=r_{\alpha}^{2} \Xi / V^{2}\left(r_{\alpha}^{2}-\chi_{\alpha}^{2}\right), C_{N L}=r_{\alpha}^{2} \Omega_{2} / V^{2}\left(r_{\alpha}^{2}-\chi_{\alpha}^{2}\right)$; yields $a_{222}^{(3)}=\left(\delta_{S} S_{N L}+\delta_{C} C_{N L}\right) \chi_{\alpha}$ and $a_{222}^{(4)}=-\left(\delta_{S} S_{N L}+\delta_{C} C_{N L}\right)$. 
The coefficients in Eq. (9 (a)) are

$$
\begin{aligned}
& a_{1}^{(1)}=a_{2}^{(1)}=a_{4}^{(1)}=0, a_{3}^{(1)}=1, a_{1}^{(2)}=a_{2}^{(2)}=a_{3}^{(2)}=0, a_{4}^{(2)}=1, \\
& a_{1}^{(3)}=-\bar{\omega}^{2} r_{\alpha}^{2} / V^{2}\left(r_{\alpha}^{2}-\chi_{\alpha}^{2}\right), \quad a_{2}^{(3)}=-\left[V^{2} C_{L}^{\alpha}\left(r_{\alpha}^{2}+(1 / 2+a) \chi_{\alpha}\right)-\pi \mu r_{\alpha}^{2} \chi_{\alpha}\left(1+\Omega_{1}\right)\right] / \pi V^{2} \mu\left(r_{\alpha}^{2}-\chi_{\alpha}^{2}\right), \\
& a_{222}^{(3)}=r_{\alpha}^{2} \chi_{\alpha}\left(\delta_{S} \Xi+\delta_{C} \Omega_{2}\right) / V^{2}\left(r_{\alpha}^{2}-\chi_{\alpha}^{2}\right), \quad a_{3}^{(3)}=\left[V C_{L}^{\alpha}\left(r_{\alpha}^{2}+(1 / 2+a) \chi_{\alpha}\right)+2 \pi \mu \bar{\omega} r_{\alpha}^{2} \zeta_{h}\right] / \pi V \mu\left(r_{\alpha}^{2}-\chi_{\alpha}^{2}\right), \\
& a_{4}^{(3)}=-\left[V r_{\alpha}^{2}\left(\pi+(1 / 2-a) C_{L}^{\alpha}\right)+V \chi_{\alpha}\left(\pi(a-1 / 2)+\left(1 / 4-a^{2}\right) C_{L}^{\alpha}\right)-4 \pi \mu r_{\alpha}^{2} \zeta_{\alpha} \chi_{\alpha}\right] / \pi V \mu\left(r_{\alpha}^{2}-\chi_{\alpha}^{2}\right), \\
& a_{1}^{(4)}=\bar{\omega}^{2} \chi_{\alpha} / V^{2}\left(r_{\alpha}^{2}-\chi_{\alpha}^{2}\right), \quad a_{2}^{(4)}=\left[V^{2} C_{L}^{\alpha}\left(\chi_{\alpha}+(1 / 2+a)\right)-\pi \mu r_{\alpha}^{2}\left(1+\Omega_{1}\right)\right] / \pi V^{2} \mu\left(r_{\alpha}^{2}-\chi_{\alpha}^{2}\right), \\
& a_{222}^{(4)}=-r_{\alpha}^{2}\left(\delta_{S} \Xi+\delta_{c} \Omega_{2}\right) / V^{2}\left(r_{\alpha}^{2}-\chi_{\alpha}^{2}\right), \quad a_{3}^{(4)}=\left[V C_{L}^{\alpha}\left(\chi_{\alpha}+(1 / 2+a)\right)+4 \pi \mu \bar{\omega} \zeta_{h} \chi_{\alpha}\right] / \pi V \mu\left(r_{\alpha}^{2}-\chi_{\alpha}^{2}\right), \\
& a_{4}^{(4)}=\left[V C_{L}^{\alpha}\left(1 / 4-a^{2}+(1 / 2-a) \chi_{\alpha}\right)+V \pi\left(a-1 / 2+\chi_{\alpha}\right)-2 \pi \mu r_{\alpha}^{2} \zeta_{\alpha}\right] / \pi V \mu\left(r_{\alpha}^{2}-\chi_{\alpha}^{2}\right) .
\end{aligned}
$$

\section{Lyapunov First Quantity (LFQ)}

An equilibrium point of a dynamical system generated by an autonomous system of ODEs is a solution that does not change with time. There are three equilibrium points founded by equating the right-hand sides of the ODEs (Eq. 9) to zero, as follows

$$
O(0,0,0,0), P\left(\frac{\sqrt{-a_{1}^{(4)} a_{2}^{(3)}+a_{1}^{(3)} a_{2}^{(4)}}\left(-a_{2}^{(4)} a_{22}^{(3)}+a_{2}^{(3)} a_{222}^{(4)}\right)}{\left(a_{1}^{(4)} a_{22}^{(3)}-a_{1}^{(3)} a_{22}^{(4)}\right)^{3 / 2}}, \frac{\sqrt{-a_{1}^{(4)} a_{2}^{(3)}+a_{1}^{(3)} a_{2}^{(4)}}}{\sqrt{a_{1}^{(4)} a_{22}^{(3)}-a_{1}^{(3)} a_{22}^{(4)}}}, 0,0\right), Q\left(\frac{\sqrt{-a_{1}^{(4)} a_{2}^{(3)}+a_{1}^{(3)} a_{2}^{(4)}}\left(a_{2}^{(4)} a_{22}^{(3)}-a_{2}^{(3)} a_{222}^{(4)}\right)}{\left(a_{1}^{(4)} a_{22}^{(3)}-a_{1}^{(3)} a_{22}^{(4)}\right)^{3 / 2}}, \frac{\sqrt{-a_{1}^{(4)} a_{2}^{(3)}+a_{1}^{(3)} a_{2}^{(4)}}}{\sqrt{a_{1}^{(4)} a_{22}^{(3)}-a_{1}^{(3)} a_{22}^{(4)}}}, 0,0\right) \text {. }
$$

The stability of typical equilibria of smooth ODEs is determined by the sign of real part of eigenvalues of the Jacobian matrix. The Jacobi matrix of equilibrium point $\boldsymbol{x}=\boldsymbol{x}_{E q}=O(0,0,0,0)$ is

$\boldsymbol{J}=\left[\begin{array}{cccc}0 & 0 & 1 & 0 \\ 0 & 0 & 0 & 1 \\ a_{1}^{(3)} & a_{2}^{(3)} & a_{3}^{(3)} & a_{4}^{(3)} \\ a_{1}^{(4)} & a_{2}^{(4)} & a_{3}^{(4)} & a_{4}^{(4)}\end{array}\right]$. Obviously, the trivial solution $\boldsymbol{x}=O(0,0,0,0)$ is the static equilibrium point of system (10). The eigenvalues of the Jacobian matrix $\boldsymbol{J}$ are the roots of the characteristic polynomial. The characteristic polynomial of $\boldsymbol{J}$ is defined as $K_{A}(\lambda)=\operatorname{det}\left(\lambda \boldsymbol{I}_{4}-\boldsymbol{J}\right)=\boldsymbol{G}$. The characteristic equation corresponding to the linearized system obtained on the flutter boundary is

$$
\lambda^{4}+p \lambda^{3}+q \lambda^{2}+r \lambda+s=0 \quad \text { where } \quad p=-\sum_{i}^{4} g_{i i}, q=\sum_{i<j}\left|\begin{array}{ll}
g_{i i} & g_{i j} \\
g_{j i} & g_{j j}
\end{array}\right|, r=-\sum_{i<j<k}\left|\begin{array}{lll}
g_{i i} & g_{i j} & g_{i k} \\
g_{j i} & g_{j j} & g_{j k} \\
g_{k i} & g_{k j} & g_{k k}
\end{array}\right|, s=\operatorname{det}(\boldsymbol{G}) \text {. }
$$

In order to investigate the character of the equilibrium point (origin $O$ ), follwoing [4] the classical Routh-Hurwitz $(\mathrm{R}-\mathrm{H})$ conditions which define the parameter bound for the stability of the system in this case can be written in the form $p>0, q>0, r>0, s>0$, and $\mathfrak{R}=p q r-s p^{2}-r^{2}>0$. Based on the Hopf bifurcation theory, if there exist a pair of pure imaginary roots and the real parts of other roots of the characteristic equation are negative, the Hopf bifurcation occurs at this point. In other words, the critical condition for flutter to occur is $\Re=0$, from which the flutter speed can be calculated; i.e. $V_{F}=\operatorname{solve}(\Re=0)$. To obtain the flutter frequency $\omega_{F}$, one may consider the solution of Eqs. (9) under the form of $x_{k}=W_{k} e^{(\hat{\omega} \tau)}$, where $\hat{\omega}=\left(\omega / V \omega_{\alpha}\right)$, the characteristic equation is $\hat{\omega}^{4}+p \hat{\omega}^{3}+q \hat{\omega}^{2}+r \hat{\omega}+s=0$; and on the flutter boundary ( $\mathfrak{R}=0)$, the roots are given by [4]:

$$
\text { 海, }= \pm i \chi, \omega_{3,4}=\varepsilon \pm i \gamma . \text { where } \chi^{2}=r / p, \varepsilon=-p / 2, \gamma^{2}=s p / r-p^{2} / 4, i=\sqrt{-1} .
$$

The stability boundaries of equilibrium points are benign (safe) and catastrophic (dangerous) [2, 4, 15]. In order to define whether the corresponding boundary of stability is benign or catastrophic, it is necessary to calculate the Lyapunov First Quantity (LFQ) $L_{1}\left(V_{F}\right)$ value on the stability boundary $\mathfrak{R}=0$. The catastrophic and benign portions of the flutter instability boundary can be determined, via determination of the sign of $L_{1}\left(V_{F}\right)$. In our case, after accomplishing some transformations and algebraic operations for $L_{1}\left(V_{F}\right)$, we may obtain the following form [4]

$$
L_{1}\left(V_{F}\right)=\frac{3 \pi}{4 \chi}\left[\left(\mathfrak{I}_{13} a_{222}^{(3)}+\mathfrak{J}_{14} a_{222}^{(4)}\right) \alpha_{21}^{3}+\left(\mathfrak{I}_{23} a_{222}^{(3)}+\mathfrak{I}_{24} a_{222}^{(4)}\right) \alpha_{22}^{3}+\left(\mathfrak{I}_{23} a_{222}^{(3)}+\mathfrak{I}_{24} a_{222}^{(4)}\right) \alpha_{21}^{2} \alpha_{22}+\left(\mathfrak{I}_{13} a_{222}^{(3)}+\mathfrak{I}_{14} a_{222}^{(4)}\right) \alpha_{21} \alpha_{22}^{2}\right]
$$




\section{Results and Discussions}

All the formulations of the aeroelastic dynamic modeling, solutions and analyses of missile control fin flying at sea level $\left(\rho_{\infty}=1.225 \mathrm{~kg} / \mathrm{m}^{3}\right)$ are performed using MATLAB ${ }^{\odot}$ and Mathematica $^{\odot}$. The configuration of the fin, considered in the present work, is shown in Fig. 5 with the geometric dimensions. Fin material is steel with the following properties: modulus of elasticity $E=2.07 \times 10^{11} \mathrm{~N} / \mathrm{m}^{2}$, Poisson's ratio $v=0.3$, and density $\rho_{m}=7850 \mathrm{~kg} / \mathrm{m}^{3}$. Fin shell thickness is $t_{\text {shell }}=2 \mathrm{~mm}$. The fin is symmetry about $x$-coordinate. Airfoil section is biconvex with $t / c=8 \%$ at

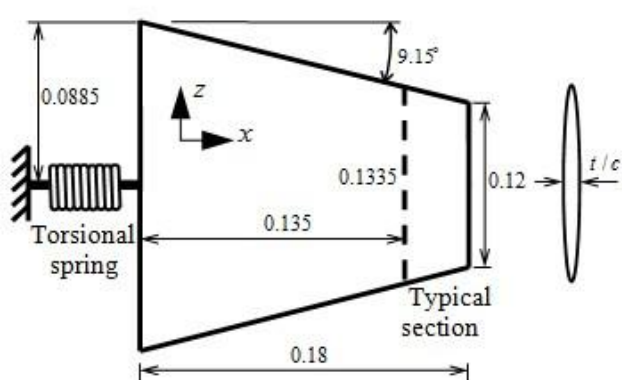

Figure 5. Fin control surface and its geometry (all dimensions are in $\mathrm{m}$ ) the root and $4 \%$ at the tip, where $t$ is the maximum thickness of the airfoil section and $c$ is the fin reference chord. Aspect and taper ratios are 2.4242 and 0.678 , respectively. Nearly $50 \%$ of the fin root, the fin is connected with a shaft. Only rotational degree-of-freedom is allowed. The other end of the shaft is completely restrained. The shaft is represented in this work by a torsional spring with coefficient $K_{x}^{\prime} \approx 800 \mathrm{~N} . \mathrm{m} / \mathrm{rad}$. which computed experimentally. The typical section is considered at section $3 / 4$ of the fin semi-span from fin root; namely $0.135 \mathrm{~m}$ (the dashed line in Fig. 5). However, to go further, the motion equations are required structure and aerodynamic parameters, mainly $\chi_{\alpha}, r_{\alpha}^{2}, a, \mu, \bar{\omega}$ and $C_{L}^{\alpha}$.

In order to demonstrate the concept of MSTMM and to build-up a model for a vibration characteristics of the fin control surface, one is often required to derive the equivalent structural beam stiffness, $E I$ and $G J$, and the position of the elastic axis. Herein the position of the elastic axis is computed from ANSYS Workbench static structure analysis module [16]. A unit torque is applied at the fin free end and the coordinates of the locus of the zero transverse deflection nodes are obtained. The elastic axis is assumed to lie along the locus of these. Consequently, the locus of these points is considered as an equivalent beam. The fin is divided into 6 elements according to the nodes on the elastic axis. The structural data such as the length, mass, mass moment of inertia about the center of gravity and the distance between elastic axis and center of gravity of these 6 elements are evaluated by 3D modeling software SPACECLAIM [17]. By separately applying a unit force (to produce bending moment $M_{b}$ ) and torque $T$ at the tip end of the elastic axis, the bending and torsional rotations $\theta_{z}$ and $\theta_{x}$ of a selected six nodes on the elastic axis can be computed. This is done through ANSYS Workbench analysis. Essentially, what is done is a back substitution in the simple beam formulas for bending and torsion, $\Delta \theta_{z}=1 / E I_{A B} \int_{A}^{B} M_{b} d s$ and $\Delta \theta_{x}=1 / G J_{A B} \int_{A}^{B} T d s$ which provide the bending and torsion stiffness, $E I_{A B}$ and $G J_{A B}$ for the element $A B$, respectively. In the view of MSTMM, the corresponding topology of the system is chain and depicted in Fig. 6 (a). The point from MSTMM analysis is to compute the in-vacuum uncoupled bending and torsional frequencies $\omega_{h}, \omega_{\alpha}$ and its ratio $\bar{\omega}$ by letting $\chi_{\alpha} \approx 0$. This chain vibrating system comprises 7 elements. Torsional spring about $x$-axis is element 1 of transfer matrix $\boldsymbol{U}_{1}=\boldsymbol{U}^{T S}$ according to Eq. (8) and the other 6 elements $(2, \cdots 7)$ are the coupled bending-torsional Euler Bernoulli beam elements $\boldsymbol{U}_{2, \ldots, 7}=\boldsymbol{U}_{2, \ldots, 7}^{C B}$ (Eq. (7)). Choosing the transfer direction from left to right, the element TE read as $\boldsymbol{Z}_{8}=\boldsymbol{U}_{7} \boldsymbol{U}_{6} \boldsymbol{U}_{5} \boldsymbol{U}_{4} \boldsymbol{U}_{3} \boldsymbol{U}_{2} \boldsymbol{U}_{1} \boldsymbol{Z}_{1}=\boldsymbol{U}^{f i n} \boldsymbol{U}_{1} \boldsymbol{Z}_{1}$. The SVs are $Z_{i}=\left[Y, \Theta_{z}, M_{z}, Q_{y}, \Theta_{x}, M_{x}\right]_{i}^{T}, i=1, \cdots 8$. The overall TE is $[6,7,9]$

$$
\boldsymbol{U}_{\text {all }} \boldsymbol{Z}_{\text {all }}=\boldsymbol{0} \text { where } \boldsymbol{U}_{\text {all }}=\left[\begin{array}{ll}
\boldsymbol{U}^{\text {fin }} \boldsymbol{U}_{1} & -\boldsymbol{I}_{6 \times 6}
\end{array}\right], \quad \boldsymbol{Z}_{\text {all }}=\left[\begin{array}{ll}
\boldsymbol{Z}_{1}^{T} & \boldsymbol{Z}_{8}^{T}
\end{array}\right]^{T} .
$$

At the boundary conditions $\left(\boldsymbol{Z}_{1}\right.$ and $\left.\boldsymbol{Z}_{8}\right)$, always half of the state variables are zero due to constraints, whereas the others are unknown. In our case, we have $\boldsymbol{Z}_{\text {all }}=\left[0,0, M_{z 1}, Q_{y 1}, 0, M_{x 1}, Y_{8}, \Theta_{z 8}, 0,0, \Theta_{x 8}, 0\right]^{T}$. Thus, Eq. (13) may be reduced to $\overline{\boldsymbol{U}}_{\text {all }} \overline{\boldsymbol{Z}}_{\text {all }}=\boldsymbol{O}$ where $\overline{\boldsymbol{Z}}_{\text {all }}=\left[M_{z 1}, Q_{y 1}, M_{x 1}, Y_{8}, \Theta_{z 8}, \Theta_{x 8}\right]^{T}$ is composed of 
the unknown state variables only, and $\overline{\boldsymbol{U}}_{\text {all }}$ is a $6 \times 6$-square matrix resulting from elimination of all columns of $\boldsymbol{U}_{\text {all }}$ associated with zeros in $\boldsymbol{Z}_{\text {all }}$. Also in this case the eigenvalue search on $\overline{\boldsymbol{U}}_{\text {all }}$ is performed with the fMin1D algorithm [8] on search interval $\omega \in[1,1000] \mathrm{rad} / \mathrm{sec}$, with grid size $N_{x 0}=5000$ and tolerance $\varepsilon=10^{-10}$. The lowest two uncoupled bending and torsion natural frequencies $\left(\omega_{h}=621.6048 \mathrm{rad} / \mathrm{sec}\right.$ and $\left.\omega_{\alpha}=671.9284 \mathrm{rad} / \mathrm{sec}\right)$ are shown in Fig. 6 (b). This yields, $\bar{\omega}=0.925106$. The positions of elastic axis and the center of gravity from the leading edge of the typical section are $0.05874 \mathrm{~m}$ and $0.058746675 \mathrm{~m}$, respectively. Typical section mass and mass moment of inertia about the elastic axis are $0.131365 \mathrm{~kg}$ and $2.1437 \times 10^{-4} \mathrm{~kg} . \mathrm{m}^{2}$, respectively. As a result, $\chi_{\alpha}=0.0001, \quad r_{\alpha}^{2}=0.36625, a=-0.12, \quad$ at sea level $\mu=42.54083$ and assume $\zeta_{h}=\zeta_{\alpha}=0$.

Computational Fluid Dynamics (CFD) approaches are becoming more popular and they are being used for complimenting the experimental studies and decreasing the number of the wind tunnel measurements. CFD analysis is carried out using CFD code ANSYS CFX over the missile fin control surface to compute the aerodynamic lift coefficient at different angles of attack and Mach number. Computations are performed on HP of 16 cores based PC with a 32GB memory. Multi block structured and unstructured meshes are generated within the computational domain as shown in Fig. 7 (a). The far-field should be placed far

(a)

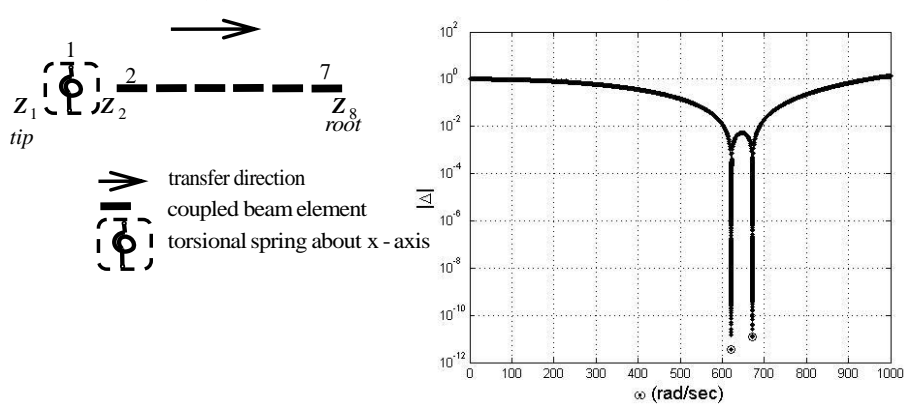

Figure 6. (a) MSTMM model of torsional spring with fin, and (b) Determinant of $\overline{\boldsymbol{U}}_{\text {all }}(\omega)$

(a)

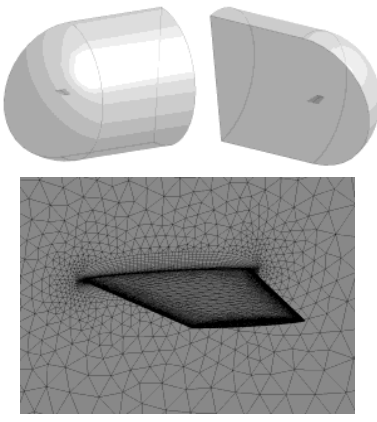

(b)

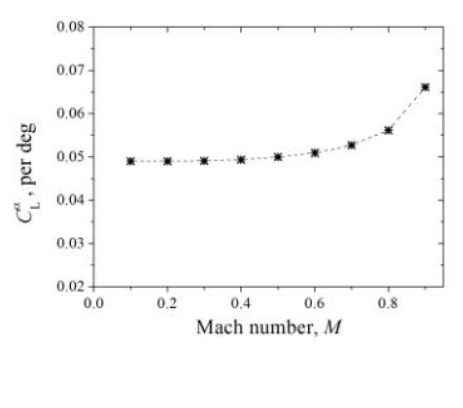

Figure 7.

(a) Far field and CFD gird generation around the fin, and (b) $C_{L}^{\alpha} v s$. Mach number enough from the fin since the free stream of infinity is defined as boundary conditions. The fin has the far-field boundary located at about $6 \mathrm{c}$ upstream away from the leading edge of the fin, about $15 \mathrm{c}$ away from the training edge downstream, and about $6 \mathrm{c}$ away from the fin in a lateral. The far field free stream condition is standard temperature and pressure $(101.325 \mathrm{kPa}, 288 \mathrm{~K})$. The air is assumed as an ideal gas and the viscosity varies with temperature in accordance with Surthland three coefficient formulas. Flow field mesh number is $905882, y^{+}<5$. A no-slip boundary condition is imposed on the fin surface, and symmetry conditions are imposed on the wall of the tunnel where the wing fixed on it. The inlet and outlet are set to be velocity and reference pressure, respectively. Reynolds Averaged Navier Stokes (RANS) based steady-state three dimensional CFD simulation are performed. Shear Stress Transport (SST) $k-\omega$ turbulent model can predict the flow separation process with higher accuracy and hence preferred for the present case of study. Figure 7 (b) is summerizing the range of $C_{L}^{\alpha} v s$. Mach number, i.e., $C_{L}^{\alpha}=(0.893 \pi \sim 1.205 \pi)$ per radian for Mach numbers $(0.1 \sim 0.9)$.

In the literature the flutter boundary is often represented by the Flutter Speed Index (the ratio of the non-dimensional flutter speed $V$ divided by the square root of mass ratio $\mu$ ). Figure 8 (a) shows the variation of Flutter Speed Index vs. flight Mach number (variation of $C_{L}^{\alpha}$ ) at sea level which obtained from the QS aerodynamic theory and CFD. It is obvious that the flutter speed decreases with the increase of the flight Mach number. As an example, a fin is flying at sea level with Mach number 0.5, Hopf bifurcation point (critical flutter velocity) is occurred as shown in Fig. 8 (b). There exist a pair of pure imaginary roots (red dots of roots 1 and 2 crossed the Img-axis) and the remaining two 

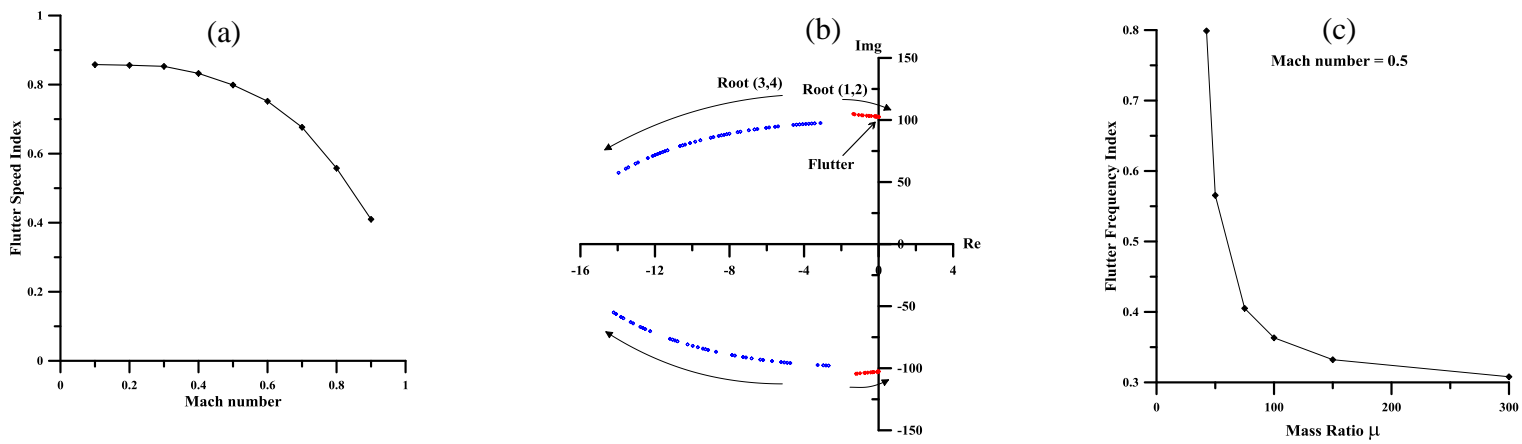

Figure 8. (a) Flutter Speed Index vs. flight Mach number at sea level, (b) Roots trajectory on the complex plane at Mach number 0.5 and sea level, and (c) Flutter Speed Index vs. mass ratio

roots (blue dots of roots 3 and 4) are complex conjugate and remain in the left-hand side of the complex plane. However, Fig. 8 (c) displayed the Flutter Speed Index decreases monotonously with

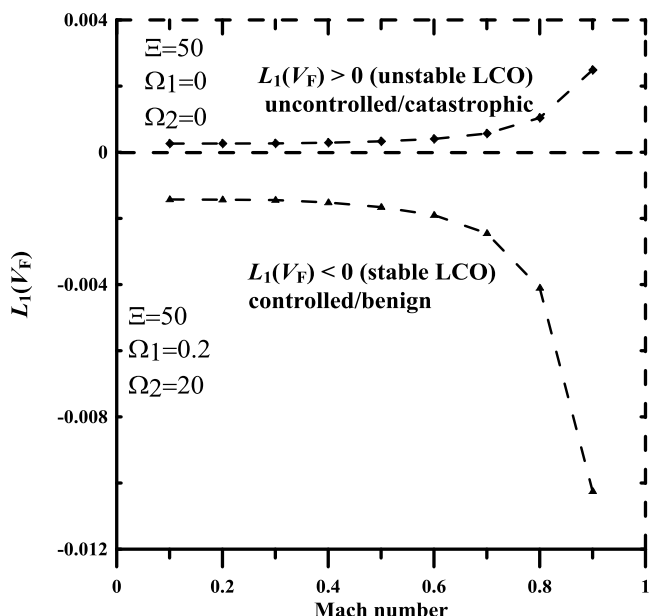

Figure 9. LFQ. Influence of structural nonlinearities on the flutter boundary for the uncontrolled/controlled cases (a)

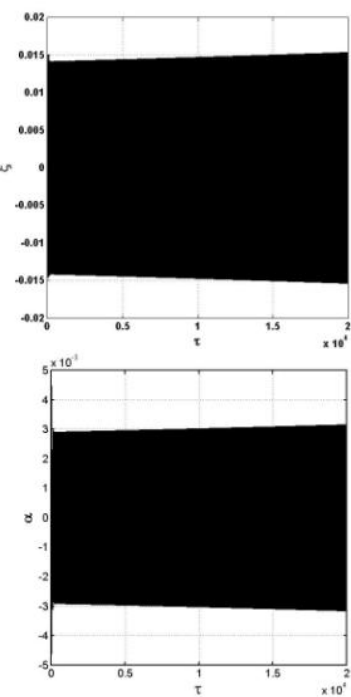

(b)

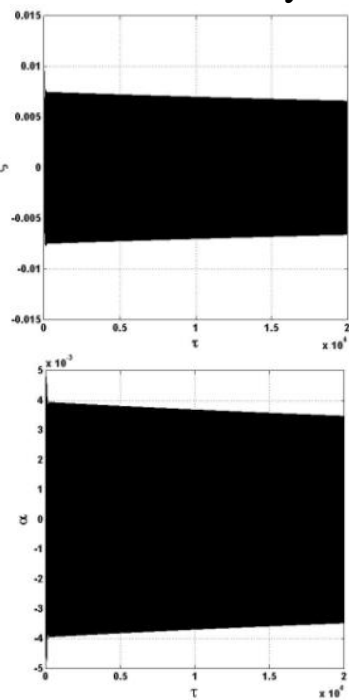

Figure 10. Transient time history solution at the boundary $\mathfrak{R}=0$ of

2-DOF airfoil section flying at sea level with Mach number 0.5 , (a) $\xi$ and $\alpha$ vs. $\tau$ (uncontrolled), and (b) $\xi$ and $\alpha$ vs. $\tau$ (controlled)

the mass ratio at Mach number 0.5.

In the plane of $\left(L_{1}\left(V_{F}\right)\right.$, Mach), Fig. 9 illustares the influence of structural nonlinearity $(\Xi=50)$ on the flutter boundary, for the uncontrolled/controlled system. The solid line identifies the case of uncontrolled $\left(\Omega_{1}=\Omega_{2}=0\right)$, whereas unstable (catastrophic) LCO occured $\left(L_{1}\left(V_{F}\right)>0\right)$. However, the system is stabilized (stable LCO) after applying the active control $\left(\Omega_{1}=0.2, \Omega_{2}=100 \Omega_{1}\right)$ represented by dashed line. To verify the theoretical results, a numerical investigation using the Runge-Kutta algorithm to the original dimensionless Eq. (9) was carried out using MATLAB ODE45. At the flutter boundary $\Re=0$, the transient motion of the airfoil flying at sea level with Mach number 0.5 and initial condition ( $\xi=0.01498, \alpha=0.00174, \xi=0, \tau=20000$ ) is depicted in Fig. 10. The bending and torsion amplitudes are growing up and no longer damped for the uncontrolled system as shown in Fig. 10 (a). Inversely, Fig. 10 (b) demonstrates the motion of the controlled system of both bending and torsion are damped. The results emerging from Figs. 9 and 10 reveals via the active control the dangerous LCO can become safe.

\section{Summary}

Using Lyapunov's first quantity theory, the character of flutter boundary and its control of a two-dimensional airfoil in the subsonic flow with cubic structural non-linearity is investigated. The flutter instability can be benign or catastrophic. The potential of active linear and nonlinear control 
issue is highlighted and increase the flutter speed and convert the dangerous boundary to the safe one.

\section{Acknowledgment}

The authors acknowledges the financial support of this research from the Natural Science Foundation of China under number 11472135, the Defense Industrial Technology Development Program under the Grants Numbers B2620132013, A2620133008 and JCKY2013606C001 and International Cooperation and Exchange Department in Nanjing University of Science and Technology.

\section{Reference}

[1] T. W. Sukut, Nonlinear aeroelastic analysis of UAVs: Deterministic and scholastic. Thesis Master of Science, Rice University, Houston, Texas, April 2012.

[2] P. Marzocca, L. Librescu, W. A. Silva, Supersonic flutter and post-flutter control of an aircraft wing section. 23rd Congress of International Council of the Aeronautical Sciences, 8-13 September, 2002, Toronto, Canada, Paper ICAS 2002-4.4.4.

[3] P. Marzocca, L. Librescu, W. A. Silva, Flutter, post flutter and control of a supersonic wing section. Journal of Guidance, Control and Dynamics 2002; 25 (5): 962-970.

[4] N. N. Bautin, The Behavior of Dynamical Systems Near the Boundaries of The Domain of Stability. $1^{\text {st }}$ Edition, Nauka, Moskva, (In Russian), 1984.

[5] X. Rui, L. Yun, Y. Lu, B. He, G. P. Wang, Transfer Matrix Method of Multibody System and its Application. $1^{\text {st }}$ Edition, Science Press, Beijing, (in Chinese), 2008.

[6] X. Rui, J. S. Zhang, Q. B. Zhou, Automatic deduction theorem of overall transfer equation of multibody system. Advances in Mechanical Engineering, 6, Article ID 378047, 2014.

[7] L. K. Abbas, Q. B. Zhou, H. Hendy, X. Rui, Transfer matrix method for determination of the natural vibration characteristics of elastically coupled launch vehicle boosters. Acta Mechanica Sinica 2015; 31 (4): 570-580.

[8] D. Bestle, L. K. Abbas, X. Rui, Recursive eigenvalue search algorithm for transfer matrix method of linear flexible multibody systems. Multibody System Dynamics 2014; 32 (4): 429-444.

[9] L. K. Abbas, M. J. Li, X. Rui, Transfer matrix method for the determination of the natural vibration characteristics of realistic thrusting launch vehicle-Part I. Mathematical Problems in Engineering, 2013; Article ID 764673, 16 pages, 2013.

[10] Encyclopedia of Aerospace Engineering. John Wiley \& Sons, Ltd, 2010.

[11] L. K. Abbas, Q. Chen, P. Marzocca, K. O'Donnell, D. Valentine, Aeroelastic behavior of lifting surfaces with free-play, and aerodynamic stiffness and damping nonlinearities. International Journal of Bifurcation and Chaos 2008; 18 (4): 1101-1126.

[12] L. K. Abbas, Q. Chen, P. Marzocca, A. Milanese, Non-linear aeroelastic investigations of store(s)-induced limit cycle oscillations. Proceedings of the Institution of Mechanical Engineers Part G Journal of Aerospace Engineering 2008; 222 (1): 63-80.

[13] L. K. Abbas, X. Rui, Free Vibration Characteristic of Multi-level Beam Based on Transfer Matrix Method of Linear Multibody Systems. Advances in Mechanical Engineering 2014; 2014 Article ID 792478, 16 pages.

[14] J. R. Banerjee, Explicit frequency equation and mode shapes of a cantilever beam coupled bending and torsion. Journal of Sound and vibration 1999; 224 (2): 267-281.

[15] S. Nikolov, V. Nedev, Stability and bifurcation behavior of an inverted pendulum with follower force. Mechanics Transport Communications 2013; 11 (2) article No. 0775: VII-1-VII-10.

[16] ANSYS, 2014. ANSYS Workbench Modeling Guide. Release 14.0, ANSYS, Inc. Canonsburg, PA, USA.

[17] SPACECLAIM. Persistent URL; http://www.spaceclaim.com. 